\title{
The use of recurrence plots and beat recordings in chronic heart failure detection
}

\author{
K. LEWENSTEIN*1 ${ }^{*}$, M. JAMROŻY ${ }^{1}$, and T.LEYKO ${ }^{2}$ \\ ${ }^{1}$ Faculty of Mechatronics, Department of Metrology and Biomedical Engineering, \\ Warsaw University of Technology, 8 A. Boboli St., 02-525 Warsaw, Poland \\ ${ }^{2}$ EMC St.Anne's Hospital in Piaseczno, Internal Medicine Department, 39 A. Mickiewicza St., 05-500 Piaseczno, Poland
}

\begin{abstract}
Chronic heart failure is a syndrome consisting in clinical symptoms that arise as a result of complications of many disease entities. According to the definition it is a condition in which, as a consequence of permanent heart dysfunction, cardiac output is reduced in relation to tissues' metabolic demand. This results in subjective symptoms or proper cardiac output is sustained over higher filling pressure of the left heart ventricle. The occurrence of disease among adults in Europe and North America is $0.4-2 \%$. Heart dysfunction detected by echocardiography appears with the frequency of $3 \%$, and in people over 60 years of age it doubles with each decade of life, reaching $10 \%$ after 80 years of age. The aim of the study is to make an acquisition of fremitus in apex beat place and quantitative evaluation of some of their features which are correlated with the advanced syndrome. We have built the apparatus for the heart signal recording and proposed the original method of its analysis based on the theory of chaos and recurrence plots. It gave us, on the group of 85 patients, sensitivity equal to 0.896 specificity equal to 0.676 and the accuracy of diagnostics of the chronic heart failure equal up to $80 \%$.
\end{abstract}

Key words: chronic heart failure, recurrence plots, nonlinear analysis, heartbeat, accelerometer, infrasound.

\section{Introduction}

Chronic heart failure (CHF) is a syndrome consisting in clinical symptoms that arise as a result of complications of many disease entities. According to the definition, CHF is a condition in which, as a consequence of permanent heart dysfunction, cardiac output is reduced in relation to tissues' metabolic demand. This results in subjective symptoms, or proper cardiac output is sustained over higher filling pressure of the left heart ventricle. The syndrome is multifactorial and due to its pathomechanisms, its clinical image may be different. The fundamental phenomenon is heart remodelling, which is a compensation mechanism. This, in unfavourable conditions, may increase pathology leading to the described syndrome. Heart remodelling lasts for many years and at some point becomes an irreversible process. When clinical symptoms are noticeable, a heart is most often in such an advanced remodelling phase that the changes are irreversible. Nowadays, patients with diagnosed heart failure are mostly people in the $6^{\text {th }}$ or $7^{\text {th }}$ decade of their life with a limited ability to perform physical activity. Frequently, the only form of treatment is transplantation. That is why detecting the risk of the disease in an early stage is so important - it can offer a chance for successful pharmacotherapy $[1,2,24]$.

The occurrence of CHF among adults in Europe and North America is $0.4-2 \%$. Heart dysfunction detected by echocardiography appears with the frequency of $3 \%$, and in people over 60 years of age it doubles with each decade of life, reaching $10 \%$ after 80 years of age. Due to the age range of the patients,

\footnotetext{
*e-mail: k.lewenstein@mchtr.pw.edu.pl
}

chronic heart failure is often called geriatric syndrome and because of its increasing frequency, we can refer to the epidemic character of this disease [1, 2].

The concept of detecting chronic heart failure described in this paper comprises the observation that the character of movements of a healthy heart in the chest differs from the character of movements of an unhealthy heart. An experienced cardiologist is able to subjectively assess if the sensed fremitus may be a symptom of pathology only by putting a hand in the place of apex beat. The aim of the study is to make an acquisition of such fremitus and quantitative evaluation of some of their features that are correlated with the advanced syndrome [3]

Heart failure is evaluated with the use of four-stage NYHA (New York Heart Association) scale. According to this scale, in the first stage a patient with no symptoms is able to perform activities causing regular effort; in fourth stage every effort causes ailments, such as tiredness, heart palpitation, and difficulties in breathing that may occur even at rest. In diagnosing CHF a significant role is played by the established sets of criteria (the so-called Framingham Criteria). If they are even partially met, it may suggest a disease.

Among laboratory analyses which can be taken into consideration in diagnosing $\mathrm{CHF}$, only checking the concentration of Brain Natriuretic Peptide (BNP) and N-terminal Pro-Brain Natriuretic Peptide (NT-proBNP) can be directly associated with the influence of damaging factors on the heart. Among other analyses aiming to determine the type and causes of heart failure, ECG, chest X-ray examination, and echocardiogram are used. The last one is currently the most important in diagnosing CHF. It enables the evaluation of the contraction activity by the assessment of the sectional and global contractility of the left 
ventricle and the measurement of the ejection fraction [1]. Other methods, based on long term heart rate variability analysis, are still not often used. A cardiologist needs to use almost all of those expensive diagnostic tools, which makes the detection of preliminary CHF state challenging. Finally, it should be stated that there is no single procedure for testing CHF. Usually almost all methods mentioned above have to be used. In our paper, we propose a new approach to diagnosing $\mathrm{CHF}$, which could be easy to use and much cheaper.

\section{Methods}

2.1. Experimental setup. The analysis of movements performed by the beating heart moving in the chest is not an easy task. While ECG signal has a characteristic course, the process of mechanical contraction of the organ is individualized and depends on its shape and the surrounding chest cavity. We can then assume that the pressure on the surface of the chest coming from the beating heart has different courses for every person. The analysis of vibrations from above the left ventricle, performed with the use of accelerometer, confirms such an observation [3]. However, people with diagnosed CHF have very accidental course, which suggests irregular organ activity.

Measurement post for the acquisition of signal from the surface of the chest, constructed in the course of the studies, consists of ECG apparatus, a set of sensors (microphone and accelerometer), preamplifiers system, synchronizing module, data acquisition card and a computer. The signal from the two mentioned sensors, amplified in the preamplifier, is sampled with the frequency of $800 \mathrm{~Hz}$ by 12 bit ADC converter of a NI USB 6008 data acquisition card. The preamplifier with a battery supply enables audio monitoring of the microphone's signal by earphones. The ECG signal received from Einthoven I and II leads and V6 Wilson precordial lead is transmitted to the computer using Bluetooth. [4]

Due to such a choice of ECG leads, we are able to determine mathematically the signals of Einthoven III leads and Goldberger leads; V6 lead receives signal mainly from the left ventricle, which is very important in CHF. ECG signals are filtered only with the use of anti-aliasing filters.

The preamplifier amplifies the signal from the accelerometer, as well as from the microphone. These signals are sampled by the measuring card; internal timer of $800 \mathrm{~Hz}$ works independently of the timer of a sampling system located in ECG apparatus. This results in synchronization problems that are solved and described in details in our previous paper [4].

Figures 1 and 2 present exemplary records of parts of the ECG, microphone and accelerometer signals taken from healthy and unhealthy persons, respectively. Even a quick look at the presented courses allows to notice their strongly stochasto-chaotic character, more apparent especially in a group of unhealthy patients. What particularly draws attention is a weak correlation of changes for the signals from the microphone and the accelerometer in unhealthy patients with heart rhythm in ECG signal. Such correlation seems to be noticeable in corresponding records taken form healthy persons. When analyzing similar records, the

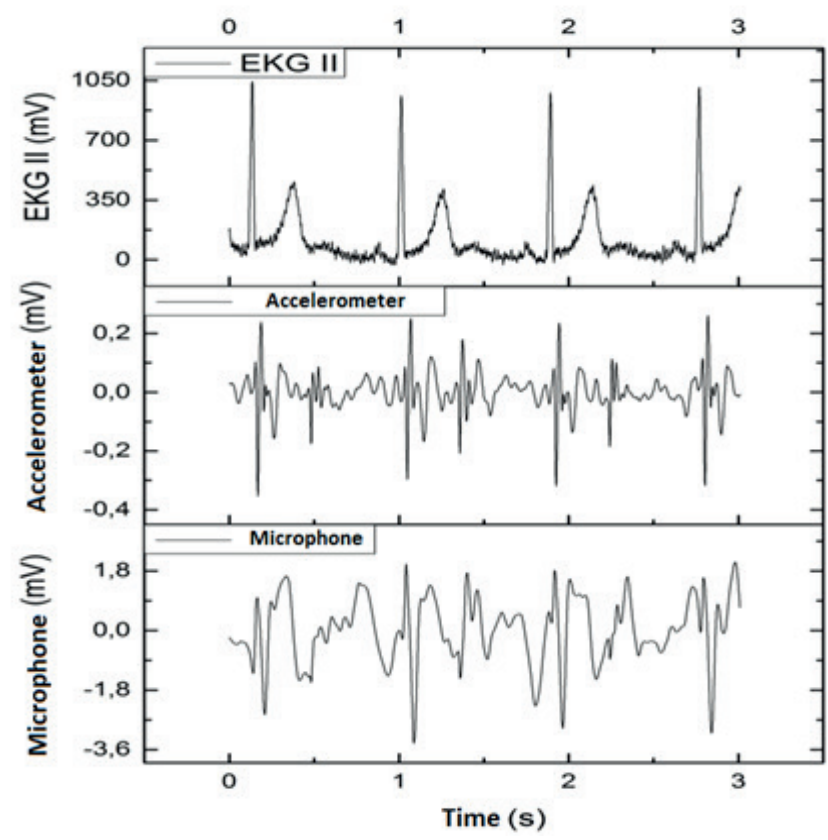

Fig. 1. Records of the parts of the signals from a healthy person

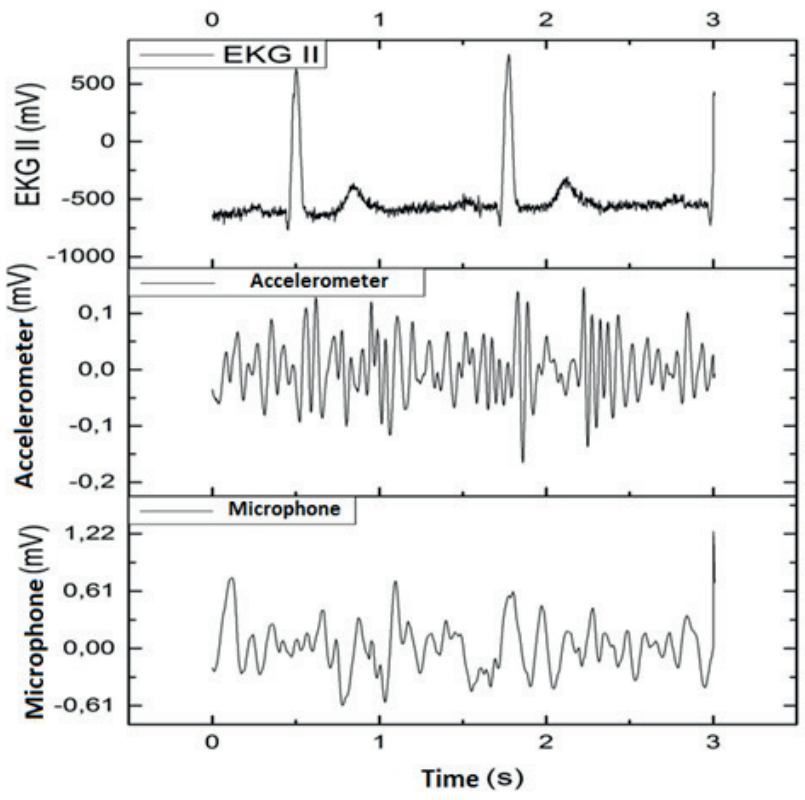

Fig. 2. Records of the parts of the signals from an unhealthy person

indicated lack of correlation seems to increase with the intensification of the disease. It altogether stipulates difficulties in the future analysis. A complicated measurement procedure impedes usage of the apparatus by medical staff. In order to avoid the influence of the human factor on the quality of the data obtained during the measurements, a specially designed, user-friendly software for an acquisition was prepared. Figure 3 presents the image of a screen - user's interface containing buttons for the acquisition process control. The record of the courses is not possible unless national identification number (PESEL) is entered and a correct system synchronization is performed. The duration of the record has been arbitrarily set to 30 seconds. When an error occurs in transmission between the apparatus and a com- 


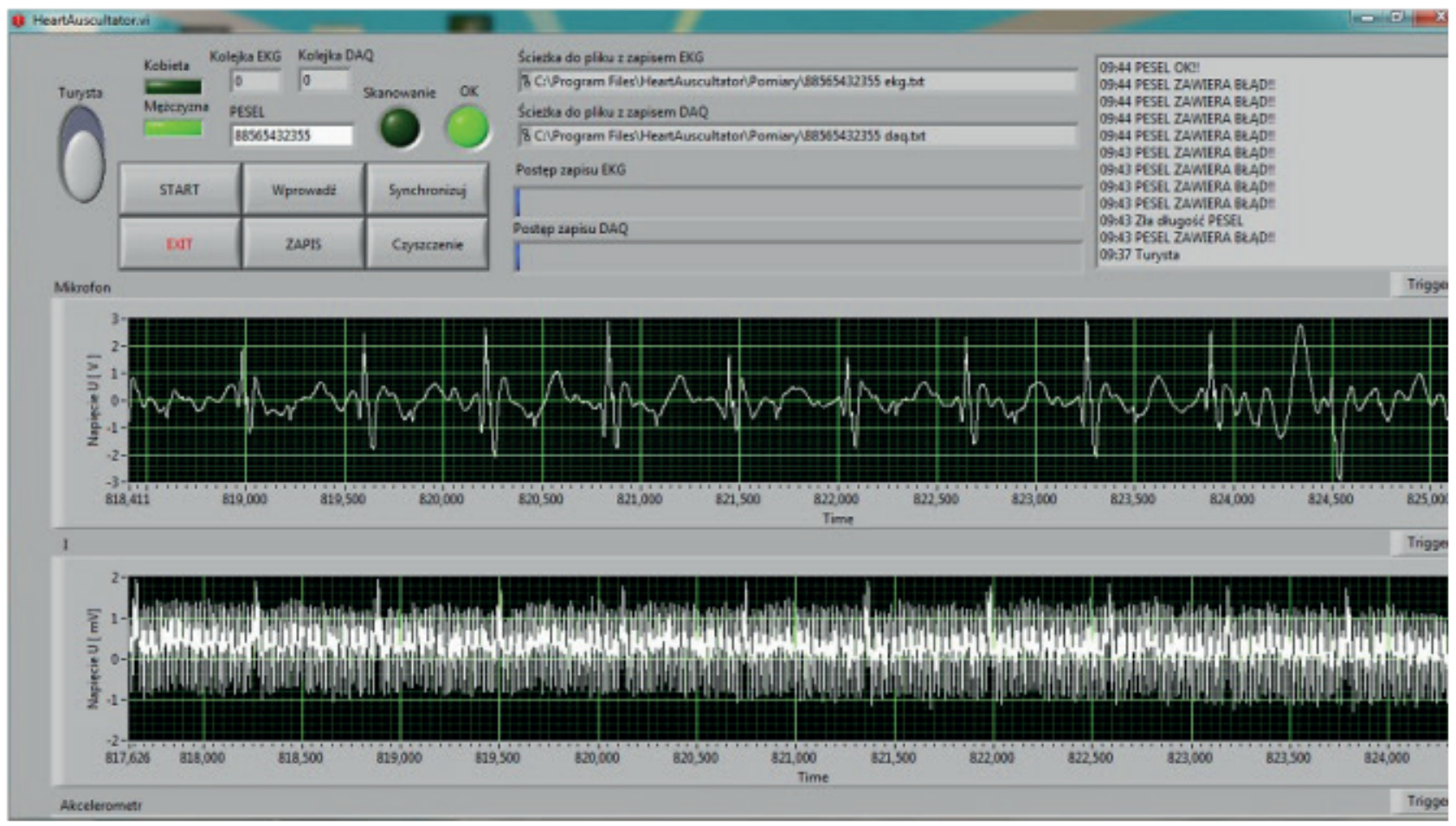

Fig. 3. User's interface of a program for examination record service

puter, the record is stopped and the announcement is shown with information about actions that have to be undertaken. The number of errors in transmission depends mainly on electromagnetic interference in the room where the examination takes place [4]. The next part of the examination is the collection of medical information that are significant in terms of CHF diagnostics. For this purpose, a dedicated application containing a form for entering relevant information about the patient was designed.

Figure 4 presents an interface of the application. The previously mentioned medical information refers to echocardio-

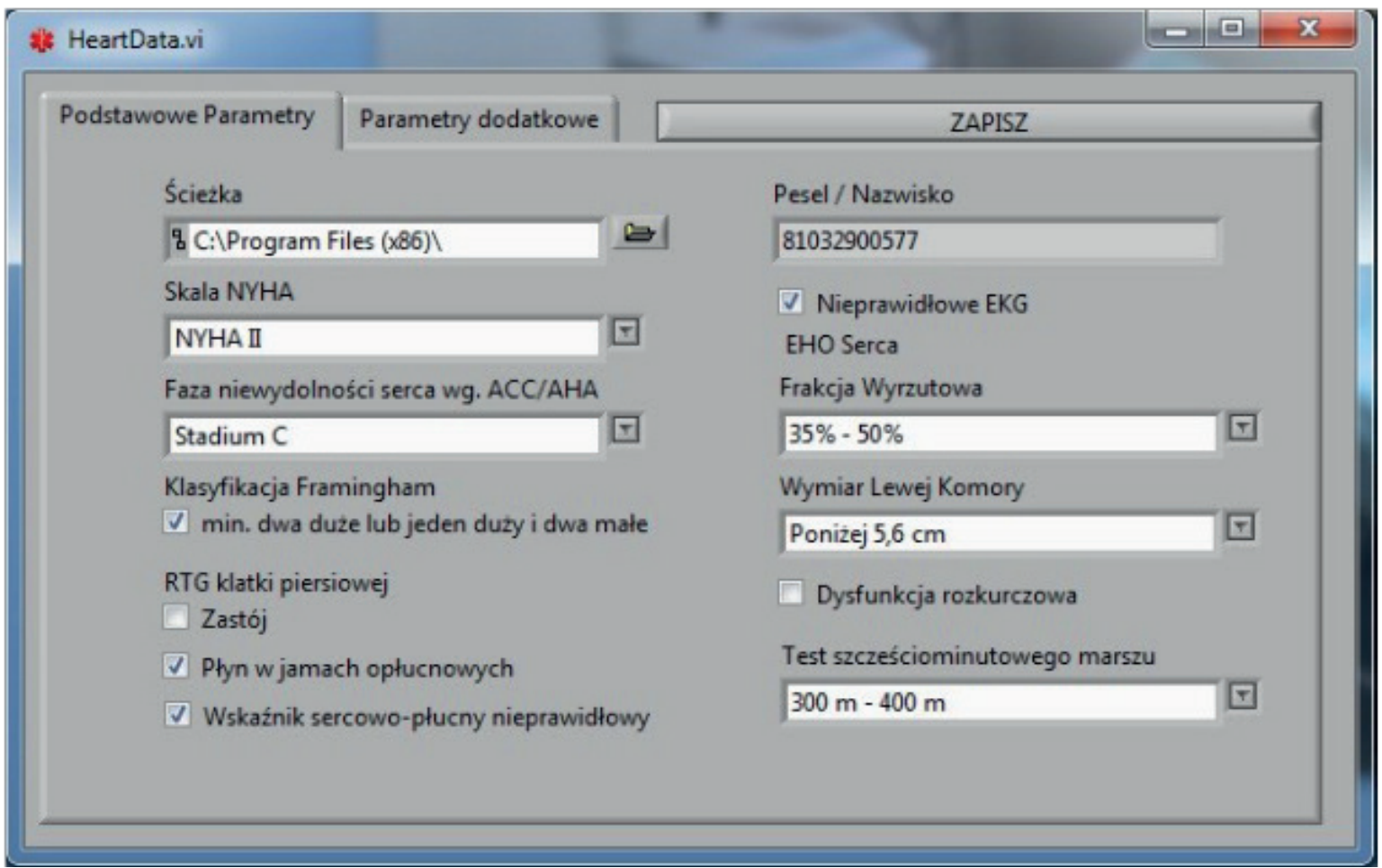

Fig. 4. Application's interface for entering medical data 
graphic examination (the dimension of the left ventricle, ejection fraction, and diastolic dysfunction), ECG test, six-minute walk test and analysis of the presence of NT-proBNP in venous blood. NT-proBNP parameter evaluates the heart's load - this quantitative information serves as a reference to the patient's condition. Its increased value demonstrates overload and is currently the most sensitive indicator of unfavourable conditions of the heartbeat. Moreover, unhealthy patients were classified according to NYHA scale and Framingham classification; the phase of their heart insufficiency was determined according to ACC/AHA guidelines. When a radiograph was available additional parameters were analyzed, such as cardiopulmonary index, presence of fluid in pleural cavity and pulmonary venostasis.

2.2. Human subjects. Patients with diagnosed chronic heart failure were included in the examined group. The only disqualifying criterion was ventricular extrasystoles or stimulation generated by the pacemaker. Volunteers with no chronic heart diseases were included in the group of healthy patients. In order to obtain a group of people with a healthy myocardium, a requirement was established that volunteers' age should be 18 to 40 years. The final group of all the examined people consisted of 85 individuals of age ranging from 21 to 87 years. Healthy people constituted a group of 37 and the unhealthy -48 .

2.3. Recurrence plots. As it was mentioned before, the analysis of the registered signals directed into CHF diagnostics aid is not an easy task. It is known that the work of the human heart is governed by many systems using different physiological phenomena, such as contraction force regulators, rhythm, coronary blood flow or blood pressure. They are very complex and non-linear, and they depend on a number of factors. The effect of their operation is an unsteadiness of signals generated by a beating heart. For the purpose of the tests of such type of dynamical systems, a non-linear analysis was implemented. This allowed for the evaluation of chaotic activity demonstrating the relation between time courses and initial conditions [5-8]. The most often analyzed parameter in this way is a heart rate variability in a 24-hours recording of ECG signal with the use of the Holter method $[9,10]$. In this paper, the registered courses are many times shorter (from 25 to 100 cycles of heart beat) and the most interesting and evaluated feature is repeatability of the mechanical course of heart contraction. It is assumed that while at rest, a healthy heart should generate pressure courses similar to each other in each cycle. Whereas in unhealthy patients, for which the pathological processes led to changes in the heart structure, this repeatability is disturbed. Observation of several dozens of heartbeat cycles should allow for evaluating this type of phenomena, despite the individual character of the pressure courses.

In the initial period of the tests, we tried to define a correlation dimension and the Lyapunov exponent calculated with the use of Nonlinear Dynamic accuracy as dominant features. However, due to relatively short recording time, we encountered problems with precise determination of the aforementioned parameters. Particularly, strong dependence of their values on the choice of the conditions during algorithms operation describing them in general disqualifies these methods as satisfactory CHF factors $[6-8,11]$.

The indicated methods of non-linear dynamics also offer more modern tools that enable testing based on the short recordings of unsteady signals, such as Recurrence Plot Method. It consists in embedding the signal in the phase space (Taken's Theory) $[8,11,12]$ :

$$
y[x(t), x(t+\Delta t), x(t+2 \Delta t), \ldots, x(t+\mathrm{m} \Delta t)]
$$

(where $\mathrm{m}$ - embedding dimension and delta $\mathrm{t}$ - time delay), and then presenting some of its features in the recurrence plot $[13-16,19]$. Both axes of the plot represent indexes of the signal samples and the coordinates of the point on the plot determine the numbers of the samples which values are evaluated. The basic recurrence plot is a dependence described by the following equation:

$$
R i, j=\Theta(\varepsilon i-\|x i-x j\|), x i \in \Re m, i, j=1 \ldots N
$$

where $\mathrm{N}$ is the length of the analyzed time course, $\mathrm{i}, \mathrm{j}$ are the coordinates on the surface, $\varepsilon \mathrm{i}$ is a radius, $\|\cdot\|$ is a norm in the metric space and $\Theta$ is a Heaviside function [16].

The procedure of generating the signal is as follows:

1. Determining delta $t$ with the use of the Average Mutual Information method.

2. Determining embedding dimension with the use of the Nearest False Neighbors method.

3. Embedding the signal in the phase space.

4. Assessing recurrence plots for the estimated $\varepsilon i$.

5. Empirically selecting $\varepsilon i$ by assigning recurrence plots until the moment of obtaining the image of the signal's structure.

6 . Visual assessment of the plot allowing for quantitative evaluation of the signal.

Exemplary recurrence plots presented in Fig. 5 (point 6) show visual evaluation of the effect of algorithm operation. It makes the analysis difficult and its result - subjective; the quality of the signal depends on the experience of the person performing the test. This problem may be omitted with the use of parameters specially calculated for this purpose. The list and the way of defining these parameters is gathered in Table 1.

The option of quantitative signal evaluation is a crucial advantage of the method allowing for processing the signal into an image and then calculating the parameters. They can become a prognostic or diagnostic information in chronic heart failure. It may also be the basis for applying computer, automated classification system [20].

When analyzing the obtained images, we can observe an increase in number of points in the recurrence plot together with the increase of ci radius. At the beginning, they appear in new places, but after reaching a certain border - they thicken the already existing structures. It means that increasing number of points makes sense only to a certain border. Depending on the features of given signal, such border is located in different places. However, regardless of the choice of percentage value of the imaging points, they present the structure of the signal dynamics. 
Table 1

Recurrence Quantification Analysis (RQA) [16-18]

\begin{tabular}{|c|c|}
\hline Indicator & Definition \\
\hline $\begin{array}{l}\text { Recurrence } \\
\text { Ratio RR }\end{array}$ & $\begin{array}{l}\text { Percent of points on the Recurrence Plot } \\
\qquad R R=\frac{1}{N^{2}} \sum_{i, j=1}^{N} R_{i, j}\end{array}$ \\
\hline Determinism DET & $\begin{array}{l}\text { Percentage of points on the lines parallel } \\
\text { to a diagonal } \\
\qquad D E T=\frac{\sum_{l=l_{\min }}^{N} l P(l)}{\sum_{i, j}^{N} R_{i, j}}\end{array}$ \\
\hline Laminarity LAM & $\begin{array}{l}\text { Percentage of points } \\
\text { on the horizontal lines } \\
L A M=\frac{\sum_{v=v_{\min }}^{N} v P(v)}{\sum_{v=1}^{N} v P(v)}\end{array}$ \\
\hline Ratio & $\begin{array}{l}\text { Relation between DET and RR } \\
\qquad R A T I O=N^{2} \frac{\sum_{l=l_{\min }}^{N} l P(l)}{\left(\sum_{l=1}^{N} l P(l)\right)^{2}}\end{array}$ \\
\hline $\begin{array}{l}\text { Average diagonal } \\
\text { length L }\end{array}$ & $\begin{array}{l}\text { Average length of a parallel line } \\
\text { to the diagonal } \\
A D L L=\frac{\sum_{l=l_{\min }}^{N} l P(l)}{\sum_{l=l_{\min }}^{N} P(l)}\end{array}$ \\
\hline Trapping time TT & $\begin{array}{l}\text { Average length of a horizontal line } \\
\qquad T T=\frac{\sum_{v=v_{\min }}^{N} v P(v)}{\sum_{v=v_{\min }}^{N} P(v)}\end{array}$ \\
\hline $\begin{array}{l}\text { The longest } \\
\text { diagonal line } \mathrm{L}_{\max }\end{array}$ & $\begin{array}{l}\text { The longest line parallel to the diagonal } \\
\qquad L_{\max }=\max \left(\left\{l_{i} ; i=1 \ldots N_{l}\right\}\right)\end{array}$ \\
\hline $\begin{array}{l}\text { The longest } \\
\text { diagonal line } \mathrm{V}_{\max }\end{array}$ & $\begin{array}{c}\text { The longest horizontal line } \\
V_{\max }=\max \left(\left\{v_{i} ; i=1 \ldots N_{v}\right\}\right)\end{array}$ \\
\hline Divergence & $\begin{array}{l}\text { Inverse of } \mathrm{L}_{\max } \\
D I V=\frac{1}{L_{\max }}\end{array}$ \\
\hline $\begin{array}{l}\text { Shannon Entropy } \\
\text { ENTR }\end{array}$ & $\begin{array}{l}\text { Shannon Entropy of probability } \\
\text { distribution of the lines parallel } \\
\text { to the diagonal } \\
\text { ENTR }=-\sum_{l=l_{\text {min }}}^{N} p(l) \ln p(l)\end{array}$ \\
\hline
\end{tabular}

- $\mathrm{N}$ - the number of trajectories in a phase space

- $\mathrm{N}_{1}$ - the number of the longest line parallel to the diagonal in the recurrence plot

- $\mathrm{N}_{\mathrm{v}}$ - the number of the longest horizontal line in the recurrence plot

- P (l), P (v) - histograms of the lengths of the lines parallel to diagonal/horizontal in the recurrence plot.
In order to make calculations with the use of a modified automatic method [21], a dedicated application was designed, in which the algorithm was implemented. The program makes calculations for the signal from the microphone, as well as from the accelerometer. It performs the analysis of all the given signals and records images and the results of RQA on a disc, in a form of a collective file with the results of all the examined patients. That makes further calculations of $\tilde{N}-$ the biggest number of diagonals parallel to the diagonal - easier.

a)

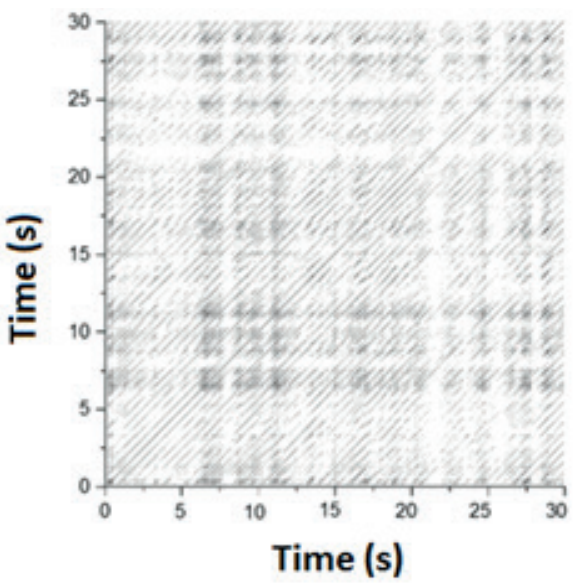

b)

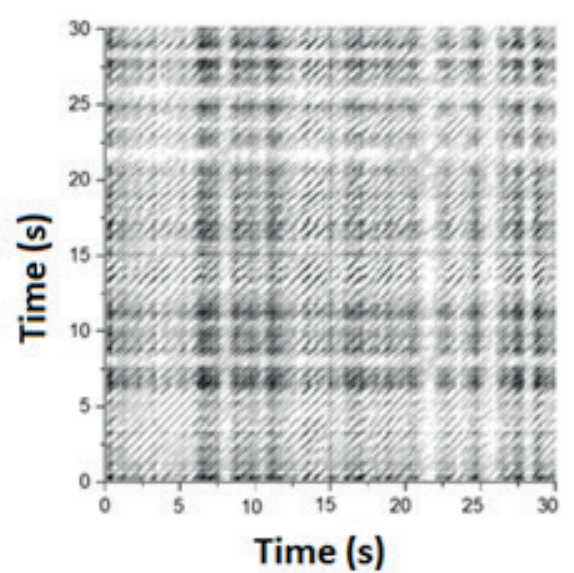

c)

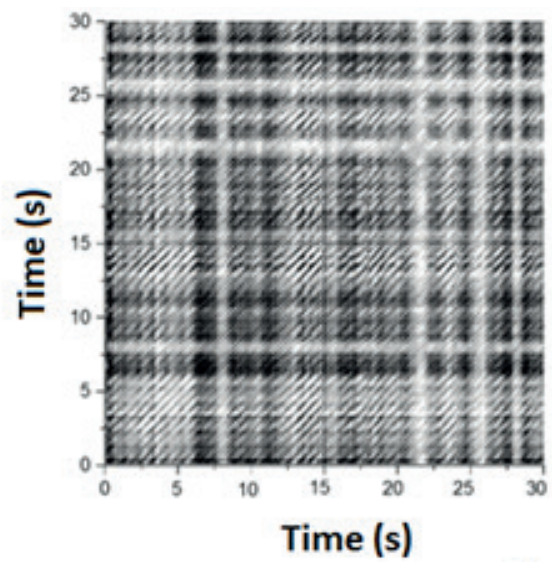

Fig. 5. Recurrence plot for ei radius covering a) $10 \%$ b) $30 \%$ c) $50 \%$ of all points of the signal 


\section{Results}

For each patient recurrence plots and RQA were calculated from microphone and accelerometer for 10,30 and $50 \%$ of points. In order to determine for which sensor and for which parameter the classification healthy-unhealthy in heart failure is the most favorable, the whole group of 85 patients was subjected to ROC (Receiver Operating Characteristic) analysis. The curves are the tool allowing for finding the same value of the parameter with optimal distinguishing values. They are the plots of relation between the sensitivity of this method and its specificity, which are defined as follows:

$$
\begin{aligned}
& \text { Sensitivity }=\frac{\mathrm{TP}}{\mathrm{TP}+\mathrm{FN}} \\
& \text { Specificity }=\frac{\mathrm{TN}}{\mathrm{TN}+\mathrm{FP}} \\
& \text { Accuracy }=\frac{\mathrm{TN}+\mathrm{TP}}{\mathrm{TN}+\mathrm{FP}+\mathrm{TP}+\mathrm{FN}},
\end{aligned}
$$

where TP and TN describe the number of the detected positive and negative cases, and FP and FN - similarly, but falsely. Vmax parameter for the microphone with $50 \%$ of points on the recurrence plot proved to be the best predictor. For the cutoff limit $V \max =573$, we obtained sensitivity equal to 0.896 and specificity equal to 0.676 ; the accuracy of diagnostics was $80 \%$. The results of the classification are presented in Table 2 .

Table 2

The table of errors for the determined predictor

\begin{tabular}{|l|c|c|}
\hline & Diagnosed as unhealthy & Diagnosed as healthy \\
\hline unhealthy & 43 & 5 \\
\hline healthy & 12 & 25 \\
\hline
\end{tabular}

ROC analysis didn't show unambiguous superiority of one sensor over the other. Despite the fact that the best distinguishability was offered by the signals from accelerometer, the next 4 highest distinguishabilities come from the accelerometer. Taking the above into consideration, we decided to make a simple classifier constructed with the use of artificial neural network modeled in MatLab environment with the use of Neural Network Toolbox ${ }^{1}$ for the five parameters, for which the distinguishability of healthy and unhealthy patients was the highest. The list of the above mentioned parameters is presented below:

- RATIO $-\mathrm{V}_{\min }=1$ for $10 \%$ of points - signal from accelerometer

- $\mathrm{LAM}-\mathrm{V}_{\min }=5$ for $40 \%$ of points - signal from accelerometer

- $\mathrm{ADLL}-\mathrm{V}_{\min }=5$ for $50 \%$ of points - signal form accelerometer

- $\mathrm{V}_{\max }$ - for $50 \%$ of points - signal from the microphone

- ENTR $-\mathrm{L}_{\min }=1$ for $30 \%$ of points - signal from accelerometer

\footnotetext{
${ }^{1}$ Software prepared by Joshua Reiss $-\mathrm{PhD}$ Thesis
}

After calibrating the data to the values included in the range $[0,1]$ we created a featured (healthy -1 , unhealthy -0 ) base of patients made of 85 records. This base was then applied in training and testing of feed-forwarded neural networks of sigmoid-shaped neurons. Treating this test as initial, the architecture of the network was chosen arbitrarily selecting 5 input neurons, 10 hidden neurons and 1 output decision neuron. The network was tested by a five group cross-validation in which the accuracy of diagnosis of $83 \%$ was obtained.

\section{Discussion}

The aim of the study was to develop a method of non-invasive acquisition of a signal coming from the heart and processing it in a way that would enable finding the features indicating a high probability of heart failure. In the authors' opinion, the aforementioned goal was achieved by processing and analyzing registered signals from accelerometer and infrasonic microphone. The presented and applied method of recurrence plots derived from the Chaos Theory is easy in its idea and offers a possibility of introducing modifications, which could increase the sensitivity and specificity of the method in the future. One or few parameters were used for diagnosing and only 85 cases were analyzed. In the situation of disposing of a more numerous base, together with a possibility to add other medical parameters to the classifying system, its effectiveness could be significantly increased. In the paper we attempted to compare the results of the signal analysis obtained by two different sensors - infrasonic microphone and accelerometer. A significant issue is population efficacy of the method, which evaluates the effect of the classification in the set acknowledging cases that were not used to teach the neural network. According to the literature, such a test was performed with the result of $83 \%$ correct diagnoses. The result is higher than for the best single classifying parameter $(80 \%)$, what indicates more complex nature of the diagnosis and at the same time encourages to optimize the list of features describing the patient, as well as the architecture or even the classifier.

\section{Conclusions}

In conclusion, it should be emphasized that the results obtained suggest a possibility of applying the method in screening examination of cardiac insufficiency. However, practical use requires conducting further tests, including cohort analysis, in order to cover a much larger number of cases. In the paper we have presented the experiment with only 85 subjects -37 healthy and 48 unhealthy (CHF) individuals were examined. For such a preliminary group we have obtained quite satisfying results - more than $80 \%$ correct detections of CHF with the use of non-optimized feature set describing patients, and also the simplest feed forward neural network classifier. There is no doubt that using a better classifying tool and a better set of features will improve the results.

Maybe a special approach for image analysis, for example the one proposed in paper [23], and the application of different 
tools - k-nearest neighbors or radial basis function neural network classifier - will lead us to a success.

Another problem that has to be solved in the future is the repeatability of the method. When there is more than one operator or the procedure is repeated several times, the measurement has to be the same. The conclusions from the results direct further works towards performing tests and further signal acquisition. It can be achieved on the basis of Bland-Altman plots [22], analyzing the relation between the result and the operator to present the signal record and repeatability of the records in time on the plot.

At the end, we would like to emphasize that we are very far from the final goal, which is clinical use of the newly proposed method. Nevertheless, we are determined to continue the research.

\section{REFERENCES}

[1] ESC Guidelines for the diagnosis and treatment of acute and chronic heart failure 2012, European Heart Journal, doi:10.1093/ eurheartk/ehs104.

[2] B.P. Griffin, E.J. Topol, G. Opolski and T. Pasierski, Cardiology Handbook Cleveland Clinic, Medipage, 2006.

[3] M. Jamroży, K. Lewenstein and T. Leyko, "Early detection of Cardiac Insufficienty", Recent Advances in Mechatronics, 407412 (2009).

[4] M. Jamroży and K. Lewenstein, "Apparatus System for Detection of Cardiac Insufficiency"., Mechatronics Recent Technological and Scientific Advances, 663-672 (2011).

[5] F. Censi, G. Calcagnini and S. Cerutti, "Proposed corrections for the quantification of coupling patterns by recurrence plots.", IEEE Trans Biomed Eng. 2004. May;51 (5):856-859.

[6] J.C. Sprott, Chaos and time-series analysis, OXFORD University Press, 2003.

[7] G.H. Schuster, Deterministic chaos: introduction, Wydawnictwo Naukowe PWN, Warszawa 1995.

[8] H.D.I. Abarbanel, Analysis of observed chaotic data, Institute for Nonlinear Science, Springer 1996.

[9] R. Maestri, G.D. Pianna, A. Accardo and P. Allegrini, "Nonlinear Indices of Heart Rate Variability in Chronic Heart Failure Patients: Redundancy and Comparative Clinical Value" J Cardiocasc Electrophysiol Vol. 18, 425-433, (2007).

[10] G.D. Addio, M. Cesarelli, M. Romano and G. Cobri, R. Maestri, "Correlation between Fractal Behavior of HRV and
Neurohormonal and Functional Idexes in Chronic Heart Failire" MEDICON 2010, IFMBE Proceedings 29, 53-56, 2010.

[11] H. Kantz and T. Schreiber, Nonlinear time series analysis, Cambridge University Press, 1997.

[12] R. Prado and M. West., Time series: modeling, computation, and inference, CRC Press, 2010.

[13] Y. Peng, Z. Sun, "Characterization of QT and RR interval series during acute myocardial ischemia by means of recurrence quantification analysis.”, Med Biol Eng Comput. 2011 Jan;49 (1):25-31.

[14] K. Klimaszewska and J.J. Zebrowski, "Detection of the type of intermittency using characteristic patterns in recurrence plots.", Phys Rev E Stat Nonlin Soft Matter Phys. 2009 Aug;80 (2 Pt 2):026214.

[15] K.C. Chua, V. Chandran, U.R. Acharya, C.M. Lim, "Computerbased analysis of cardiac state using entropies, recurrence plots and Poincare geometry", J Med Eng Technol. 2008, Jul-Aug;32 (4):263-72.

[16] http://www.recurrence-plot.tk/

[17] A. Arcentales, B.F. Giraldo, P. Caminal and S. Benito, A Voss, "Recurrence quantification analysis of heart rate variability and respiratory flow series in patients on weaning trials", Conf Proc IEEE Eng Med Biol Soc. 2011:2724-7.

[18] C.D. Nguyen, S.J. Wilson and S. Crozier, "Automated quantification of the synchrogram by recurrenceplot analysis", IEEE Trans Biomed Eng. 2012, Apr;59 (4):946-55.

[19] M. Mohebbi, H. Ghassemian and B. Mohammadzadeh, "Structures of the Recurrence Plot of Heart Rate Variability Signal as a Tool for Predicting the Onset of Paroxysmal Atrial Fibrillation", J Med Signals Sens. May-Aug; 1 (2): 113-121 (2011).

[20] K. Lewenstein, Artificial neural networks in the diagnostics of coronary artery diseases based on ECG exercise tests, Oficyna Wydaw. PW, Warsaw 2002.

[21] M. Jamrozy, K. Lewenstein and T. Leyko, "Automatic analysis of recurrence plot for the needs of the analysis of infrasonic signals from the human heart", Mechatronics 2013 - Recent Technological and Scientific Advances, Springer 2013.

[22] G.D. Altman and J.M. Bland, "Measurement in medicine: the analysis of method comparison studies", The Statistician 32: 307-317, 1983.

[23] R. Tadeusiewicz and M. Ogiela, "Structural approach to medical image understanding", Bull. Pol. Ac.: Tech. 52 (2), 131-139 (2004).

[24] M. Tycler, M. Turzowa et al., "Use of body surface maps for model based assessment of local pathological changes on the heart", Bull. Pol. Ac.: Tech. 53 (3), 207-215 (2005). 